\title{
Analyst
}

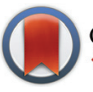

CrossMark

\& click for updates

Cite this: Analyst, 2015, 140, 1804

Received 8th December 2014,

Accepted 28th January 2015

DOI: $10.1039 / c 4 a n 02248 c$

www.rsc.org/analyst

\section{En route to traceable reference standards for surface group quantifications by XPS, NMR and fluorescence spectroscopy $\dagger$}

\author{
Andreas Hennig, ${ }^{\star a, b}$ Paul M. Dietrich, ${ }^{* a}$ Felix Hemmann, ${ }^{a}$ Thomas Thiele, ${ }^{c}$ \\ Heike Borcherding, ${ }^{\mathrm{C}}$ Angelika Hoffmann, ${ }^{\text {a }}$ Uwe Schedler, ${ }^{\mathrm{C}}$ Christian Jäger, ${ }^{\text {a }}$ \\ Ute Resch-Genger*a and Wolfgang E. S. Unger ${ }^{a}$
}

The fluorine content of polymer particles labelled with 2,2,2-trifluoroethylamine was reliably quantified with overlapping sensitivity ranges by XPS and solid-state NMR. This provides a first step towards reference materials for the metrological traceability of surface group quantifications. The extension of this concept to fluorescence spectroscopy is illustrated.

The controlled functionalization of surfaces has moved into the focus of many material and life scientists as this paves the way for applications in biosensing, drug delivery, implantation medicine, separation sciences, optoelectronics, and solar energy conversion. ${ }^{1-3}$ The precise knowledge of the chemical nature, areic group density, and spatial distribution of surface functional groups is thus key for the broad application of existing and rational design of improved functional materials as well as for the public acceptance of new nanotechnology-based materials. It is similarly relevant for a reliable quality control during material fabrication and processing and thus, for their reproducible large-scale production. Consequently, numerous analytical methods have been used for the characterization of functionalized surfaces. ${ }^{2,4-7}$

Despite the overall importance of a reliable and quantitative surface analysis, we recently noted that surface group quantification methods are usually not mutually validated..$^{5-7}$ In fact, even the application of more than one surface group quantification method to the same material is not always common practice. This is particularly severe for surface quantification methods relying on the use of chemical derivatization agents. Therein, a quantitative coupling yield is often presumed,

\footnotetext{
${ }^{a}$ BAM Federal Institute for Materials Research and Testing, Richard-WillstaetterStrasse 11, 12203 Berlin, Germany. E-mail: paul.dietrich@bam.de, ute.resch@bam.de

${ }^{b}$ Jacobs University Bremen, School of Engineering and Science, Campus Ring 1, 28759 Bremen, Germany. E-mail: a.hennig@jacobs-university.de

${ }^{c}$ PolyAn GmbH, Rudolf-Baschant-Strasse 2, 13086 Berlin, Germany

$\dagger$ Electronic supplementary information (ESI) available: Experimental details, details on quantification, and supplementary figures. See DOI: 10.1039/ c4an02248c
}

which may not always hold true..$^{5-9}$ In addition, certain surface functional group quantification methods such as X-ray photoelectron spectroscopy (XPS) have a limited information depth and require elaborated data analysis based on theoretical models to address layered structures, especially on curved surfaces. ${ }^{7}$ However, also these quantification models have so far only rarely been experimentally verified. ${ }^{10}$

A standard reference material applicable to various surface functional group quantification methods is thus highly desirable. This would allow mutual method validation as well as experimental testing of theoretical quantification models. A particular challenge in this endeavour is to provide overlapping sensitivity ranges for the various methods, which all have different limits regarding the highest and lowest concentrations that can be reliably detected. Moreover, certain surface analysis methods (e.g. XPS) are mainly applied to planar functionalized surfaces rather than to particles, while others (e.g. NMR) are commonly applied to bulk materials rather than to surface analysis. ${ }^{4}$

Herein, we now present the first step towards the development of such a reference material. We have previously extensively characterized polymer particles composed of a poly(methyl methacrylate) (PMMA) core with a grafted shell of poly(acrylic acid) (PAA), ${ }^{5,6}$ including a detailed characterization by XPS before and after labelling with 2,2,2-trifluoroethylamine (TFEA). ${ }^{7}$ The fluorine content of these particles was now additionally determined by ${ }^{19} \mathrm{~F}$ solid-state NMR, which provides an unprecedented link in the traceability chain between XPS and NMR. Furthermore, we illustrate that the detection sensitivity by ${ }^{19} \mathrm{~F}$ NMR is also sufficient to provide overlapping sensitivity ranges with fluorescence spectroscopy. We thus disclose herein a strategy towards reference materials for the surface functional group quantification by NMR, XPS, and fluorescence spectroscopy.

We selected four different PMMA particle batches with varying amounts of surface PAA $\left(0,35,99\right.$, and $946 \mu \mathrm{mol} \mathrm{g}^{-1}$, referred to as P0, P35, P99, and P946). The number of surface $\mathrm{COOH}$ groups was previously determined by conductometry, ${ }^{5-7}$ 
and the amount of surface-grafted PAA on P946 was now confirmed with solid-state ${ }^{13} \mathrm{C}$ NMR by comparing the integrated peak areas of the carboxy region and the methyl group. This gave PAA surface densities of $(1600 \pm 1000) \mu \mathrm{mol} \mathrm{g}^{-1}$ for P946, which agree well with the results from conductometry within the stated measurement uncertainties.

The different PAA-functionalized particles were analysed by XPS, which gave the elemental composition within the first

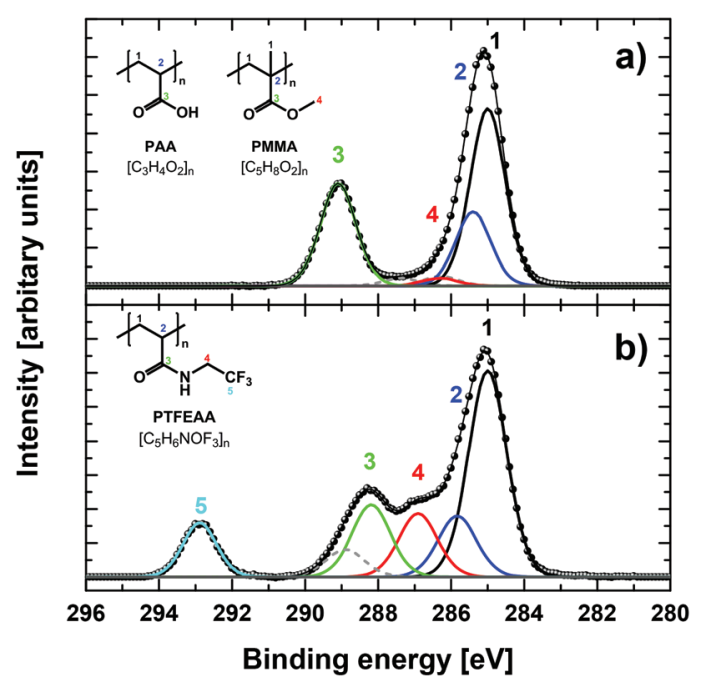

Fig. 1 High-resolution $C$ 1s core-level spectra of PMMA/PAA particles P946 (a) before and (b) after chemical derivatization with TFEA. Additional components originating from poly( $N$-vinylpyrrolidone) encapsulated in the PMMA core are shown as dashed lines in grey.

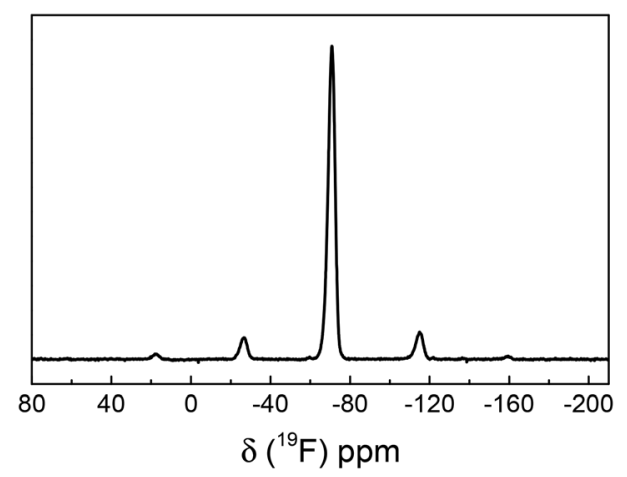

Fig. 2 Solid-state ${ }^{19} \mathrm{~F}$ MAS NMR spectrum of TFEA-labelled P946.
$10 \mathrm{~nm}$ of the surface (XPS information depth). ${ }^{7}$ The survey spectra as well as the high-resolution $\mathrm{C}$ 1s and O 1s XP spectra of the different PMMA/PAA microparticles were all perfectly consistent with the varying amounts of surface-grafted PAA of the unlabelled particles obtained by our previous studies. ${ }^{5-7}$ Successful covalent labelling with TFEA according to a previously established protocol (Scheme S1a in ESI†), ${ }^{5}$ was confirmed by significantly altered photoelectron spectra, in particular by the appearance of a new peak in the survey and core-level spectra corresponding to the $\mathrm{CF}_{3}$ group ( $c f$. Fig. 1a and $\mathrm{b})^{7}$

Successful covalent surface labelling with TFEA was also independently confirmed by solid-state ${ }^{19} \mathrm{~F}$ NMR. All NMR spectra showed a peak at $\delta=(-70.7 \pm 0.3)$ ppm with MAS spinning side bands, which is the typical peak position of the $\mathrm{CF}_{3}$ group of trifluoroethylamide (Fig. 2). This is a striking result, since it demonstrates that even the particles with the lowest amount of surface-grafted PAA could be successfully analysed by ${ }^{19} \mathrm{~F}$ NMR within reasonable time. This is due to the high gyromagnetic ratio and natural abundance of the ${ }^{19} \mathrm{~F}$ nucleus, which is only outperformed by ${ }^{1} \mathrm{H}$ NMR in terms of its relative sensitivity. The latter is, however, inapplicable to routine surface group quantifications because of the omnipresence of hydrogen atoms and a comparably small frequency range of typical ${ }^{1} \mathrm{H}$ NMR resonances in combination with line broadening in solid-state NMR.

After characterization of the TFEA-labelled particles, the amount of surface-bound TFEA was quantified by XPS and solid-state ${ }^{19}$ F NMR spectroscopy (Table 1). By XPS, the fraction of TFEA-labelled $\mathrm{COOH}$ groups was determined by comparing the integrated peak areas of carbon and fluorine atoms in the survey spectra. ${ }^{711}$ This gave coupling yields of $32 \%$ for P35, $54 \%$ for P99, and $47 \%$ for P946, respectively. Subsequently, the quantification of surface-bound TFEA by solid-state ${ }^{19} \mathrm{~F}$ NMR was performed. Typically, known amounts of sample and standard are mixed and the integrated peak areas (including the areas of the MAS side bands) of the sample are referenced to the signal derived from a known amount of the ${ }^{19} \mathrm{~F}$ intensity standard. ${ }^{12}$ This is, however, in the present case not feasible, because only a very low fraction of the sample is TFEA-labelled, namely just the surface groups. The required amount of standard, i.e., 4-(trifluoromethyl) benzoic acid, is thus too small to reliably afford a homogeneous mixture of sample and reference. As an alternative, reference and sample were measured consecutively in different rotors under the same experimental

Table 1 TFEA coupling yields of microparticles

\begin{tabular}{|c|c|c|c|c|c|c|}
\hline & {$[\mathrm{COOH}]^{a}\left(\mu \mathrm{mol} \mathrm{g}^{-1}\right)$} & \multicolumn{3}{|l|}{$\mathrm{XPS}^{b}$} & \multicolumn{2}{|l|}{${ }^{19} \mathrm{~F}-\mathrm{NMR}$} \\
\hline P99 & 99 & $20.8 \pm 1.3$ & $54 \pm 3$ & $53 \pm 3$ & $26 \pm 1$ & $26 \pm 1$ \\
\hline P946 & 946 & $19.9 \pm 0.4$ & $47 \pm 2$ & $443 \pm 24$ & $39 \pm 2$ & $367 \pm 18$ \\
\hline
\end{tabular}

${ }^{a}$ Total number of surface $\mathrm{COOH}$ groups as determined by conductometry (uncertainty $c a .9 \%$, see ref. 5 ). ${ }^{b}$ Taken from ref. 7 (uncertainty $10 \%$ ).

${ }^{c}$ Coupling yield calculated using 4 -(trifluoromethyl) benzoic acid as reference. 
conditions, particularly using the same receiver gain. This procedure was repeated several times to confirm the spectrometer stability. The results indicated a maximum deviation between individual measurements of about $1 \%$. This analysis gave TFEA coupling yields of $30 \%, 26 \%$, and $39 \%$ for P35, P99, and P946, respectively.

The coupling yields determined by XPS and solid-state ${ }^{19} \mathrm{~F}$ NMR are in good agreement for P35 and P946, and they are significantly higher than those previously reported for the fluorescein derivative FL-A (Chart S1 in ESI $\dagger$ ) and adamantylmethylamine (both around 5\%). ${ }^{5,6}$ This is probably due to the smaller size of TFEA and to a much lower $\mathrm{p} K_{\mathrm{a}}$ of the amino group of TFEA $\left(\mathrm{p} K_{\mathrm{a}}=5.7\right)$ compared to aliphatic amines $\left(\mathrm{p} K_{\mathrm{a}} \sim 10\right)$ leading to a significantly increased reactant concentration, yet they do not reach the maximum coupling yield of $50 \%$ claimed for PAA. ${ }^{13}$ The results for P99 obtained by XPS, however, exceed this value and are also significantly higher than those determined by NMR and should consequently be treated with caution. We believe that several factors contribute to this discrepancy, which are all related to the intermediary thickness of the TFEA-labelled PAA layer on randomly distributed, spherical $P 99$ particles. For example, in case that the thickness of the probed layer is smaller than the XPS information depth, the PMMA core additionally contributes to the amount of carbon used for quantification of TFEA-labelled $\mathrm{COOH}$ groups. In addition, a recent XPS depth profiling study has revealed an increased concentration of the labelling reagent within the top few nanometers of grafted polymers, which is indiscernible in bulk measurements like NMR but clearly influences the XPS results. ${ }^{5,14}$ For this combination of factors, common quantification models should usually not be applied, and we are currently developing alternative quantification models to address this issue. Nonetheless, even when including P99, the relative error of the average of all coupling yields $(38 \pm 10 \%)$ is still in accordance with our previous surface quantification method comparisons with much larger sample sets. ${ }^{5,6}$

Most important, our results clearly demonstrate the potential of fluorine as an attractive heteroatom for the development of reference materials for traceable surface functional group quantifications. The utilization of our TFEA-functionalized polymer particles now allows the combined use of XPS and solid-state ${ }^{19} \mathrm{~F}$ NMR spectroscopy for surface group quantifications on the same material. Although a combination of XPS and NMR has been used complementary to characterize particle-based sample materials such as hybrid organic-inorganic materials and nanodiamonds, ${ }^{15}$ it has, to the best of our knowledge, neither been used to mutually validate both methods nor for establishing a metrological traceability chain. With the identification of a very pure and suitable reference standard containing both ${ }^{19} \mathrm{~F}$ and ${ }^{1} \mathrm{H}$, XPS could even be traced back to a certified NMR reference standard and thus to the SI unit mole via the quantitative method solid-state ${ }^{19} \mathrm{~F}$ NMR as shown in Fig. 3a.

We have previously prepared and comprehensively characterized the same selection of polymer particles labelled

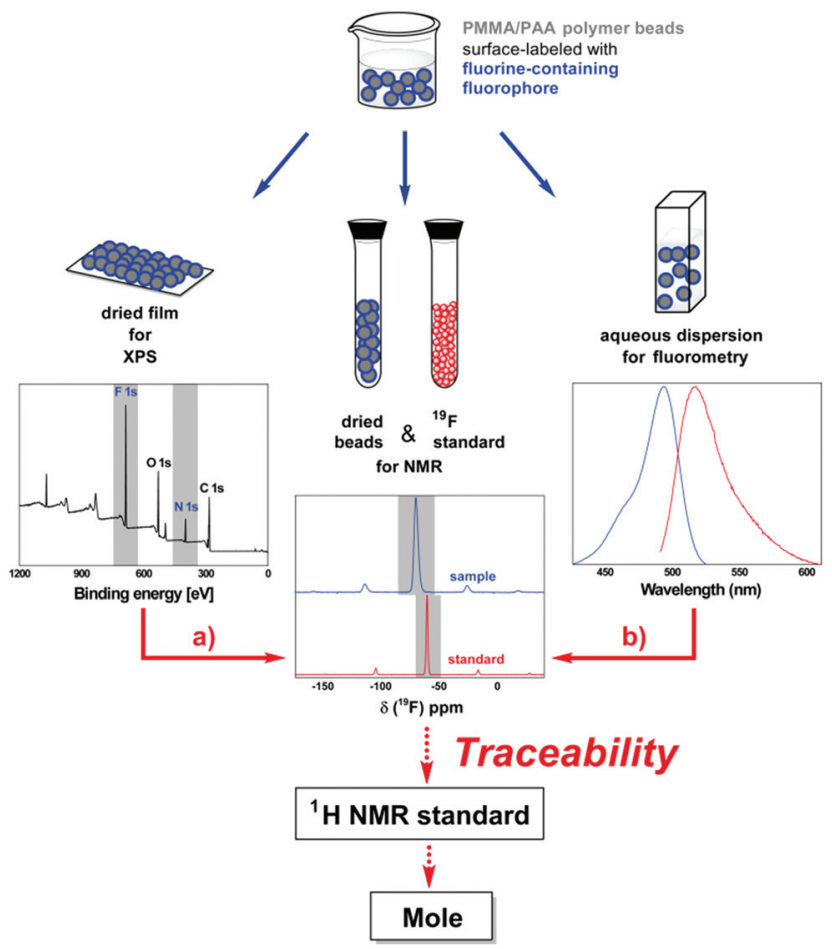

Fig. 3 Traceability chain for surface group quantification, linking measurements (blue arrows) of (a) XPS and (b) fluorometry to quantitative solid-state ${ }^{19} \mathrm{~F}$ NMR (solid red arrows). The use of a certified NMR reference standard containing both ${ }^{19} \mathrm{~F}$ and ${ }^{1} \mathrm{H}$ provides the link to the $\mathrm{SI}$ unit mole (dotted red arrows).

with a mixture of the fluorescein derivative FL-A and varying amounts of $\mathrm{H}_{2} \mathrm{~N}-\mathrm{CH}_{2}\left(\mathrm{OCH}_{2}\right)_{3}-\mathrm{CO}_{2} \mathrm{H}$ (added to prevent particle aggregation). ${ }^{5}$ The $\mathrm{P} 946$ particles contain the highest practically relevant amounts of surface-bound fluorophores, which have been successfully quantified by absolute fluorometry and the surface concentrations of fluorophores were in the range of 0.42 to $6.3 \mu \mathrm{mol} \mathrm{g}{ }^{-1}$. However, a tentative recording of XPS spectra did not show any peaks assignable to the thiourea group of FL-A. Even evaporating a drop of a solution containing suspended unlabelled particles and unbound FL-A, that gave a spot containing the polymer particles and a FL-A concentration of $c a .13 \mu \mathrm{mol} \mathrm{g}^{-1}$, showed the sulfur peak for the thiourea group only with an inacceptable signal-to-noise ratio. This clearly indicates that the combination of maximum achievable surface concentration of fluorophores and sensitivity of XPS for sulfur is insufficient, while detection of fluorine would be unproblematic in this concentration range by XPS and even by NMR. This is not only ascribed to the higher content of heteroatoms in TFEA (three fluorine atoms) compared to FL-A (one sulfur atom), but also to the enhanced sensitivity of XPS for fluorine. ${ }^{16}$ This is consistent with previous results on plasma-chemically modified polypropylene films, in which the nitrogen atom (which has a XPS sensitivity comparable to fluorine) of the fluorescent label dansyl was qualitatively detected by fluorescence and XPS. ${ }^{17}$ 
Overall, we believe that fluorine-containing fluorophores will present ideal candidates to additionally link fluorescence spectroscopy to the traceability chain now established for XPS and NMR (Fig. 3b). Fortunately, fluorine is included in several fluorescent dyes. ${ }^{18}$ In fact, we recently reported the determination of particle-encapsulated fluorophores by absolute fluorometry and ${ }^{19} \mathrm{~F}$ NMR. ${ }^{12}$ In the case of surface-bound fluorophores, the sensitivity ranges could additionally be finetuned by adjusting the number of fluorine atoms per fluorophore through the use of fluoroalkyl linkers with varying lengths and numbers of fluorine atoms. The resulting increase in number of fluorine atoms per molecule would even enable decreasing the amount of surface fluorophores to concentrations, at which fluorescence self-quenching does not apply. Thereby, we could not only link XPS and NMR to absolute fluorometry, ${ }^{12}$ which accounts for varying fluorescence quantum yields, but also to the much more widely used relative fluorometry. ${ }^{5}$

\section{Conclusions}

We demonstrated that fluorine on the surface of polymer microparticles labelled with TFEA could be detected by XPS and solid-state ${ }^{19} \mathrm{~F}$ NMR with overlapping detection ranges. Consistent coupling yields of the small reporter TFEA were determined by XPS and quantitative solid-state ${ }^{19} \mathrm{~F}$ NMR spectroscopy establishing a very important step towards the metrological traceability of surface group quantifications. Furthermore, we disclosed a strategy to include fluorometry as an additional surface quantification method with comparable detection ranges and currently pursue the identification of fluorine-containing fluorophores with suitable spectroscopic, photophysical and chemical properties. ${ }^{5,6 c, 18}$ Such fluorinecontaining fluorescent dyes would be key for a prospective reference material and could additionally be utilized as a chemical derivatization reagent for a reliable and traceable quantification of chemically addressable surface functional groups. $^{5,8,9}$

\section{Notes and references}

$\$$ The large uncertainty for P946 is attributed to the signal-to-noise ratio of the ${ }^{13} \mathrm{C}$ MAS NMR spectra, i.e. the $(\mathrm{COOH}+\mathrm{COOMe}) / \mathrm{C}^{-\mathrm{CH}_{3}}$ ratio was $1.16 \pm 0.10$ for P946 and subtracting the value of 1.00 for the unmodified PMMA core gives $0.16 \pm 0.10$ moles of surface $\mathrm{COOH}$ per mole of PMMA monomer in the core.

1 (a) K. Chatterjee, S. Sarkar, K. Jagajjanani Rao and S. Paria, Adv. Colloid Interface Sci., 2014, 209, 8-39; (b) S. Rödiger, C. Liebsch, C. Schmidt, W. Lehmann, U. Resch-Genger, U. Schedler and P. Schierack, Microchim. Acta, 2014, 181, 1151-1168; (c) R. A. Sperling and W. J. Parak, Ther. Innovation Regul. Sci., 2013, 47, 1333-1383; (d) C. Xu and S. Sun, Adv. Drug Delivery Rev., 2013, 65, 732-743; (e) C. T. Adkins, J. N. Dobish, S. Brown and E. Harth, ACS Macro Lett., 2013, 2, 710-714; $(f)$ J. M. Montenegro,
V. Grazu, A. Sukhanova, S. Agarwal, J. M. de la Fuente, I. Nabiev, A. Greiner and W. J. Parak, Adv. Drug Delivery Rev., 2013, 65, 677-688; ( $g$ ) K. Hoffmann, T. Behnke, D. Drescher, J. Kneipp and U. Resch-Genger, ACS Nano, 2013, 7, 6674-6684; (h) K. Saha, S. S. Agasti, C. Kim, X. Li and V. M. Rotello, Chem. Rev., 2012, 112, 2739-2779; (i) J. M. Baumes, J. J. Gassensmith, J. Giblin, J. J. Lee, A. G. White, W. J. Culligan, W. M. Leevy, M. Kuno and B. D. Smith, Nat. Chem., 2010, 2, 1025-1030; (j) A. I. Abdelrahman, S. Dai, S. C. Thickett, O. Ornatsky, D. Bandura, V. Baranov and M. A. Winnik, J. Am. Chem. Soc., 2009, 131, 15276-15283; (k) L. Barner, Adv. Mater., 2009, 21, 2547-2553; (l) U. Resch-Genger, M. Grabolle, S. Cavaliere-Jaricot, R. Nitschke and T. Nann, Nat. Methods, 2008, 5, 763-775.

2 (a) K. E. Sapsford, W. R. Algar, L. Berti, K. B. Gemmill, B. J. Casey, E. Oh, M. H. Stewart and I. L. Medintz, Chem. Rev., 2013, 113, 1904-2074.

3 (a) J. E. Gagner, S. Shrivastava, X. Qian, J. S. Dordick and R. W. Siegel, J. Phys. Chem. Lett., 2012, 3, 3149-3158; (b) N. B. Shah, G. M. Vercellotti, J. G. White, A. Fegan, C. R. Wagner and J. C. Bischof, Mol. Pharm., 2012, 9, 21462155; (c) K. E. Sapsford, K. M. Tyner, B. J. Dair, J. R. Deschamps and I. L. Medintz, Anal. Chem., 2011, 83, 4453-4488; (d) D. F. Moyano and V. M. Rotello, Langmuir, 2011, 27, 10376-10385; (e) S. J. Tan, N. R. Jana, S. J. Gao, P. K. Patra and J. Y. Ying, Chem. Mater., 2010, 22, 22392247.

4 D. R. Baer, M. H. Engelhard, G. E. Johnson, J. Laskin, J. Lai, K. Mueller, P. Munusamy, S. Thevuthasan, H. Wang, N. Washton, A. Elder, B. L. Baisch, A. Karakoti, S. V. N. T. Kuchibhatla and D. Moon, J. Vac. Sci. Technol., A, 2013, 31, 050820.

5 A. Hennig, H. Borcherding, C. Jaeger, S. Hatami, C. Würth, A. Hoffmann, K. Hoffmann, T. Thiele, U. Schedler and U. Resch-Genger, J. Am. Chem. Soc., 2012, 134, 8268-8276.

6 (a) A. Hennig, A. Hoffmann, H. Borcherding, T. Thiele, U. Schedler and U. Resch-Genger, Chem. Commun., 2011, 47, 7842-7844; (b) A. Hennig, A. Hoffmann, H. Borcherding, T. Thiele, U. Schedler and U. ReschGenger, Anal. Chem., 2011, 83, 4970-4974; (c) A. Hennig, S. Hatami, M. Spieles and U. Resch-Genger, Photochem. Photobiol. Sci., 2013, 12, 729-737.

7 P. M. Dietrich, A. Hennig, M. Holzweber, T. Thiele, H. Borcherding, A. Lippitz, U. Schedler, U. Resch-Genger and W. E. S. Unger, J. Phys. Chem. C, 2014, 118, 2039320404.

8 (a) B. Panella, A. Vargas, D. Ferri and A. Baiker, Chem. Mater., 2009, 21, 4316-4322; (b) S. Huang, R. Joso, A. Fuchs, L. Barner and S. V. Smith, Chem. Mater., 2008, 20, 5375-5380; (c) Y. Xing, N. Dementev and E. Borguet, Curr. Opin. Solid State Mater. Sci., 2007, 11, 86-91; (d) K. Hoffmann, M. Mix, U. Resch-Genger and J. F. Friedrich, Langmuir, 2007, 23, 8411-8416; (e) K. Qi, Q. Ma, E. E. Remsen, C. G. Clark Jr. and K. L. Wooley, J. Am. Chem. Soc., 2004, 126, 6599-6607; (f) V. B. Ivanov, 
J. Behnisch, A. Holländer, F. Mehdorn and H. Zimmermann, Surf. Interface Anal., 1996, 24, 257-262.

9 (a) A. Chilkoti and B. D. Ratner, in Surface Characterization of Advanced Polymers, ed. L. Sabbatini and P. G. Zambonin, Wiley VCH, 1993, p. 221; (b) A. Chilkoti and B. D. Ratner, Surf. Interface Anal., 1991, 17, 567-574; (c) A. Chilkoti, B. D. Ratner and D. Briggs, Chem. Mater., 1991, 3, 51-61; (d) C. D. Batich, Appl. Surf. Sci., 1988, 32, 57-73; (e) H. Kondo and Y. Nishida, Bull. Chem. Soc. Jpn., 2007, 80, 1405-1412; $(f)$ V. I. Povstugar, S. S. Mikhailova and A. A. Shakov, J. Anal. Chem., 2000, 55, 405-416; (g) J. Vickerman and I. S. Gilmore, Surface Analysis - The Principal Techniques, Wiley, 2nd edn, 2009.

10 (a) P.-L. Girard-Lauriault, T. Gross, A. Lippitz and W. E. S. Unger, Anal. Chem., 2012, 84, 5984-5991; (b) A. G. Shard, J. Phys. Chem. C, 2012, 116, 16806-16813; (c) G. Zorn, S. R. Dave, X. Gao and D. G. Castner, Anal. Chem., 2011, 83, 866-873; (d) S. Techane, D. R. Baer and D. G. Castner, Anal. Chem., 2011, 83, 6704-6712; (e) M. Mohai and I. Bertóti, Surf. Interface Anal., 2012, 44, 1130-1134; $(f)$ M. Mohai and I. Bertóti, Surf. Interface Anal., 2004, 36, 805-808.

11 T. Gross, F. Pippig, B. Merz, R. Merz, U. Vohrer, R. Mix, H. Steffen, W. Bremser and W. E. S. Unger, Plasma Processes Polym., 2010, 7, 494-503.
12 A. Huber, T. Behnke, C. Wurth, C. Jaeger and U. ReschGenger, Anal. Chem., 2012, 84, 3654-3661.

13 (a) E. R. Bissell and M. J. Finger, J. Org. Chem., 1959, 24, 1256-1259; (b) G. T. Hermanson, Bioconjugate Techniques, Academic Press (Elsevier), London, 2nd revised edn, 2008, p. 1202; (c) N. Nakajima and Y. Ikada, Bioconjugate Chem., 1995, 6, 123-130.

14 R. Barbey, V. Laporte, S. Alnabulsi and H.-A. Klok, Macromolecules, 2013, 46, 6151-6158.

15 (a) H. Virieux, M. Le Troedec, A. Cros-Gagneux, W.-S. Ojo, F. Delpech, C. Nayral, H. Martinez and B. Chaudret, J. Am. Chem. Soc., 2012, 134, 19701-19708; (b) O. Shenderova, A. M. Panich, S. Moseenkov, S. C. Hens, V. Kuznetsov and H.-M. Vieth, J. Phys. Chem. C, 2011, 115, 19005-19011.

16 J. H. Scofield, J. Electron Spectrosc. Relat. Phenom., 1976, 8, 129-137.

17 K. Hoffmann, U. Resch-Genger, R. Mix and J. F. Friedrich, J. Fluoresc., 2006, 16, 441-448.

18 (a) B. R. Renikuntla, H. C. Rose, J. Eldo, A. S. Waggoner and B. A. Armitage, Org. Lett., 2004, 6, 909-912; (b) A. Loudet and K. Burgess, Chem. Rev., 2007, 107, 48914932; (c) M. Hecht, T. Fischer, P. Dietrich, W. Kraus, A. B. Descalzo, W. E. S. Unger and K. Rurack, ChemistryOpen, 2013, 2, 25-38. 